DOI https://doi.org/10.30525/978-9934-26-019-3-4

\title{
APPLICATION OF THE PROJECTION-ITERATIVE VERSION OF THE LOCAL VARIATIONS METHOD TO STABILITY PROBLEMS FOR SPHERICAL SHELLS UNDER LOCALIZED LOADS
}

\author{
Hart E. L., Hudramovich V. S.
}

\section{INTRODUCTION}

Many problems of mechanics are considered in a variational formulation and for their study the apparatus and methods of functional analysis are used in appropriately selected functional spaces. This approach allows us to identify common patterns inherent in wide classes of extremal problems, to create and explore general methods for solving them. Note that for most variational problems in the mechanics of a deformable solid, the use of the quadratic functional of potential energy is characteristic.

The local variations method (LVM), developed by F.L Chernousko and his coauthors [7], refers to effective numerical methods for solving variational problems. This method is a variant of the methods of variations in the phase space developed in the works of N. N. Moiseev, which are based on a change in the phase components of the trajectory [7].

The LVM has a number of advantages compared to other numerical methods. It makes it easy to take into account restrictions on the desired functions, the arbitraries of the shape of the region, and other features. In mechanics of a deformable solid, the LVM is effective for substantially inhomogeneous stressed states. This method allows one to consider different types of loading of thin-walled systems and boundary conditions, the different structure of such systems (for shells and plates variability of rigidity, cuts). It should be noted, however, that when solving certain classes of problems, for example, variational, which reduce to linear boundary-value problems, the LVM requires more calculation time to achieve a given accuracy than finite-difference methods and variational methods of the Ritz type. The search for ways to eliminate this drawback led to the idea of developing more effective schemes for its implementation based on the ideology of projection- 
iterative methods $[3,12]$, which can significantly reduce the computer calculation time.

The effectiveness of projection-iterative schemes for implementing the finite difference method and finite element method for solving a wide range of problems in the theory of elasticity and plasticity was shown in $[8,9]$. The development of a projection-iterative modification of yet another numerical method for solving variational problems - LVM, is of undoubted interest. We point out that the issues of reducing the machine time for the LVM account were given attention earlier [5].

\section{The main provisions of the projection-iterative LVM-modification}

Consider the problem of finding in a domain with a boundary a function delivering a minimum to the quadratic functional

$$
F=\iint_{G} f\left(x, y, u, u_{x}^{\prime}, u_{y}^{\prime}\right) d x d y=\iint_{G}\left\{S\left(\frac{\partial u}{\partial x}\right)^{2}+Q\left(\frac{\partial u}{\partial y}\right)^{2}+R u^{2}+2 T u\right\} d x d y
$$

and satisfying the boundary condition

$$
u=g(x, y),(x, y) \in \Gamma \text {. }
$$

Here $S(x, y), Q(x, y), R(x, y), T(x, y), g(x, y)$ are the given continuously differentiable functions of independent variables.

To solve the problem (1), (2) we apply the projection-iterative modification of the local variations method. To do this, we write the original problem in the form:

$$
F(u) \rightarrow \text { inf, } u \in \Omega,
$$

where $\Omega$ is some set of real Hilbert space $H ; F(u)$ - bounded below by $\Omega$ the functional $\left(\inf _{u \in \Omega} F(u)=F^{*}>-\infty\right)$. In particular, $H=L_{2}(\Omega) ; \Omega$ is the set of functions from $L_{2}(\Omega)$ satisfying the boundary conditions (2).

Let us state the main idea of the projection-iterative modification of the LVM given in $[1,2,10]$. The initial functional $F(u)$ defined on some set $\Omega$ of Hilbert space $H$ is approximated by a sequence of simpler functionals $\tilde{F}_{n}\left(\tilde{u}_{n}\right)$ defined on some sets $\tilde{\Omega}_{n}$ of spaces $\tilde{H}_{n}$ isomorphic to subspaces $H_{n}$ of the original space $H\left(u_{n}=\Phi_{n}^{-1} \tilde{u}_{n}, u_{n} \in H_{n}, \quad \tilde{u}_{n} \in \tilde{H}_{n}\right.$, 
$\Phi_{n}$ is an operator that performs a one-to-one correspondence between elements of subspaces $H_{n}$ and $\tilde{H}_{n}$ ). To minimize the obtained sequence of functionals $\tilde{F}_{n}\left(\tilde{u}_{n}\right)$, the LVM is applied in such a way that, starting from a certain number $n=N$ (corresponding to a rough partition of the domain $G$ ), a sufficiently small number $h_{n}$, and some initial approximation $\tilde{u}_{n, i j}^{(k)}\left(k=0, i=\overline{0, N_{1}}, j=\overline{0, N_{2}}\right)$, the values $\tilde{u}_{n, i j}^{(k)}$ at all internal points $\left(x_{i}, y_{j}\right)$ of the domain $G$ are varied sequentially in some order by adding or subtracting the value $h_{n}$. The variation does not continue until complete convergence (i.e., when further fragmentation of the step $h_{n}$ does not reduce the value of the functional), but until the functional is almost reduced and the solution $\tilde{u}_{n, i j}^{(k)}\left(k=0,1, \ldots, k_{n}\right)$ changes in a small number of points, i.e. $\left|\tilde{F}_{n}\left(\tilde{u}_{i j}^{(k)}\right)-\tilde{F}_{n}\left(\tilde{u}_{i j}^{(k+1)}\right)\right|<\varepsilon_{n}$, $\left.\mid \tilde{u}_{n, i j}^{(k)}-\tilde{u}_{n, j}^{(k+1)}\right) \mid<\delta_{n}$, with the exception of $(i, j) \in I_{n}$, where $I_{n}$ is a fixed set of power indices $<<\left(N_{1}-1\right) \times\left(N_{2}-1\right)$. Further, the found value $\tilde{u}_{n, i j}^{(k)}$ is interpolated into a smaller partition and is used as an initial approximation to minimize the following functional $\tilde{F}_{n+1}\left(\tilde{u}_{n+1}\right)$. The process continues until the iterations completely converge at some small step $h_{n}$ and the condition

$$
\left|\tilde{F}_{n}\left(\tilde{u}_{n}^{\left(k_{n}\right)}\right)-\tilde{F}_{n+1}\left(\tilde{u}_{n+1}^{\left(k_{n+1}\right)}\right)\right|<\varepsilon,
$$

where $\varepsilon$ is the specified accuracy of the calculations.

\section{Application of the projection-iterative LVM-modification} to the solution of the variational problem with quadratic functional

As in [7], we divide the plane $O x y$ into equal rectangular cells with parallel lines $x=x_{0}+i \Delta x_{n}, \quad y=y_{0}+j \Delta y_{n}$. Here $\Delta x_{n}>0, \Delta y_{n}>0$ are fairly small numbers, $i, j=0, \pm 1, \pm 2, \ldots ; n$ is a certain natural number corresponding to the step number of the process of partitioning the region $G$. We choose the points $x_{0}, y_{0}$ and the natural numbers $N_{1}^{(n)}, N_{2}^{(n)}$ so that for given values $\Delta x_{n}, \Delta y_{n}$ the region $G+\Gamma$ is contained in a rectangle $\bar{G}$ : 


$$
\bar{G}=\left\{(x, y) \in R_{2}: \begin{array}{c}
x_{0} \leq x \leq x_{N_{1}^{(n)}}=x_{0}+N_{1}^{(n)} \Delta x_{n} ; \\
y_{0} \leq y \leq y_{N_{2}^{(n)}}=y_{0}+N_{2}^{(n)} \Delta y_{n}
\end{array}\right\}
$$

Denote by $P_{i j}$ the intersection points of lines (cell vertices) with coordinates $x_{i}=x_{0}+i \Delta x_{n}, \quad y_{j}=y_{0}+j \Delta y_{n} \quad\left(i=\overline{0, N_{1}^{(n)}} ; j=\overline{0, N_{2}^{(n)}}\right) . \quad W e$ will consider a point $P_{i j}$ to be internal if it, together with the four cells whose vertex it is, lies in a closed region $G+\Gamma$. The remaining points $P_{i j}$ lying in the region $G+\Gamma$ will be considered boundary, and the points outside the region $G+\Gamma-$ external. Put $u_{n, i j}=u\left(x_{i}, y_{j}\right)$. We transfer condition (2) from the contour $\Gamma$ to the boundary points, assuming for them $u_{n, i j}=g\left(\xi_{i}, \eta_{j}\right)$, where $\left(\xi_{i}, \eta_{j}\right)$ is the $\Gamma$-contour point, in a sense close to $\left(x_{i}, y_{j}\right)$. For example, you can take, as $\left(\xi_{i}, \eta_{j}\right)$ the contour point closest to the point $\left(x_{i}, y_{j}\right)$ or define it by the drift condition along one of the coordinate axes: $\xi_{i}=x_{i}$ or $\eta_{j}=y_{j}$.

The integral (2) is approximately replaced by the sum of the integrals over the cells entirely belonging to the domain $G+\Gamma$ :

$$
F \approx \tilde{F}_{n}=\sum_{i j} \tilde{F}_{n, i j}
$$

where $\tilde{F}_{n, i j}$ is the approximate value of the integral over the cell with the vertices $P_{i j}, \quad P_{i+1, j}, \quad P_{i, j+1}, \quad P_{i+1, j+1}$; the index $n$ indicates the correspondence of the obtained domain $G$ discretization expression with steps $\Delta x=\Delta x_{n}, \Delta y=\Delta y_{n}$.

We will search for values $u_{i j}$ for all internal points $P_{i j}$ of the region $G$ that deliver a minimum to expression (5). Thus, from the original problem (1), (2) we proceed to the sequence of tasks of minimizing the function of many variables

$$
\tilde{F}_{n}\left(\tilde{u}_{n}\right) \rightarrow \inf , \quad \tilde{u}_{n} \in \tilde{\Omega}_{n} \subset \tilde{H}_{n},
$$


where $\quad \tilde{\Omega}_{n}=\Phi_{n} \Omega_{n}, \quad \Phi_{n} \quad$ is the linear operator of one-to-one correspondence between spaces $H_{n}$ and $\tilde{H}_{n} \quad\left(\Phi_{n} u_{n}=\tilde{u}_{n}, \Phi_{n}^{-1} \tilde{u}_{n}=u_{n}\right)$; $\tilde{H}_{n}$ is a subspace isomorphic . Suppose that a sequence of subspaces is extremely dense in, i.e.

$$
\forall u \in H \quad \exists\left\{u_{n}\right\} \in H_{n}:\left\|u-u_{n}\right\| \rightarrow 0 \quad \text { при } n \rightarrow \infty .
$$

Starting with some sufficiently coarse partition corresponding to a certain number, we will look for values that minimize the sum (1) using the method of successive approximations. As a zeroth approximation, we take any set of numbers satisfying the constraints (2). A good choice of the zeroth approximation can significantly accelerate the convergence of the method, therefore, here we should take into account the available a priori information on the proposed solution (qualitative, physical, and other considerations).

\section{The convergence theorem}

We formulate a convergence theorem for the proposed modification.

Theorem. Let a functional $F(u)$ of the form (1), bounded below, be given on the set $\Omega$ of a real Hilbert space $H$. Suppose that a sequence of subspaces $H_{n}$ is extremely dense in $H$. The sequence of approximations $\left\{\Phi_{n}^{-1} \tilde{u}_{n}^{\left(k_{n}\right)}\right\}$ to the minimum point of functional (1) is constructed according to the rule:

$$
\tilde{u}_{n}^{(k+1)}=\left\{\tilde{u}_{n, 11}^{(k+1)}, \tilde{u}_{n, 12}^{(k+1)}, \tilde{u}_{n, 1 l}^{(k+1)}, \tilde{u}_{n, 21}^{(k+1)}, \ldots, \tilde{u}_{n, m l}^{(k+1)}\right\},
$$

where $\tilde{u}_{n, i j}^{(k+1)}\left(i=\overline{1, m}, j=\overline{1, l} ; \quad k=0,1, \ldots, k_{n}-1\right)$ are determined from condition (4); $k_{n}$ is the number of iterations to approach the minimum point of the functional $\tilde{F}_{n}\left(\tilde{u}_{n}\right) ; \tilde{u}_{n+1}^{(0)}$ is a new initial approximation to the minimum point of the functional $\tilde{F}_{n+1}\left(\tilde{u}_{n+1}\right)$ obtained by interpolation:

$$
\begin{gathered}
\tilde{u}_{n+1}^{(0)}=\Phi_{n+1} \Phi_{n}^{-1} \tilde{u}_{n}^{\left(k_{n}\right)} \\
\left(k=0,1, \ldots, k_{n}-1 ; n=1,2, \ldots ; \quad \tilde{u}_{1}^{(0)} \in \tilde{\Omega}_{1}\right) .
\end{gathered}
$$


Then the projection-iterative sequence $\left\{\Phi_{n}^{-1} \tilde{u}_{n}^{\left(k_{n}\right)}\right\}$ constructed by formulas (7), (8) converges to the extremal $u^{*}$ of the functional $F(u)$ on $\Omega$.

The proof of the theorem is given in [10].

Note that, in contrast to the LVM proposed in [7], the projection-iterative modification of this method allows one to significantly save computer time, since it does not require at each step to achieve complete convergence in $h$ for fixed $\Delta x, \Delta y$. In addition, in practice, the final values $\Delta x, \Delta y, h$ are not known in advance, and it seems more expedient to use the stop criterion (4), which also allows one to significantly reduce the counting time, ensuring the given accuracy of the solution.

It should also be noted that, in contrast to the LVM modification described in [5] and using the LVM scheme with a variable variation step [7], the projection-iterative modification of the LVM is simpler to implement and requires significantly less computation time on a PC, which does not affect on the quality of the solution $[1,2,10]$.

\section{Local stability of spherical shells}

Let us consider the application of the proposed LVM-modification to the problems of local stability of spherical shells under a substantially inhomogeneous stress state caused by local edge loading. These tasks are important for the construction of space rocket, antenna technology, etc. $[4,11]$. Local loading is carried out through dies, the behavior of which is described by different models (Winkler, Vlasov, Muravsky, etc.), and is perceived by a force ring that reinforces the edge of the shell. Such a load transfer scheme simulates the transverse loading of the spherical diaphragms of the extended shell structures of rocketry, mirror antennas, and radiant energy concentrators.

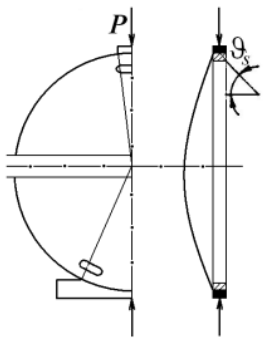

a

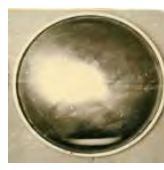

b

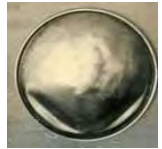

$\mathrm{C}$

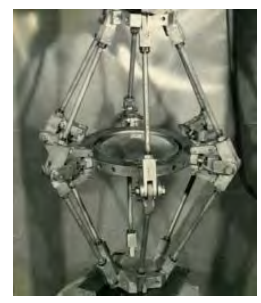

d

Fig. 1. The loading scheme (a), the waveforms $(b, c)$ and type of experimental apparatus (d) 
In Fig. 1 shows the loading schemes, the waveforms of local buckling, and the type of experimental apparatus.

Contact pressure is variable along the contact areas at various degrees of its localization, which depends on the size of the contact areas, the parameters of the stiffness of the dies and design. This determines the form of wave formation. In Fig. 1a,b for a small contact area - one dent, in Fig. 1,a,c for an extended area - two dents (in this case, the contact pressure is concentrated in the edge zones of the dies). For hard dies, a contact breakdown can occur (the shell and the ring move away from the stamp) with the formation of discrete contact pads of various lengths. Fig. 1b,c show the tested shells made of aluminum-magnesium alloy AMG6M, manufactured by explosive stamping (with monitoring the deviation of the shell surface from spherical). In Fig. 1d shows a developed device that allows testing shells under loading by different stamp systems for various local loading schemes (including the scheme shown in Fig. 1a).

We point out that in the theoretical consideration of contact interaction, it is necessary to solve a peculiar "double" contact problem: for a shell and a ring with a determination of the forces of their interaction and a ring loaded with these forces with a stamp. Methods for solving such problems were developed in $[4,11]$.

To describe the behavior of spherical shells, we use the relations of the nonlinear theory of shallow shells [6]. The system of equations defining the critical loads of the shells is equivalent to the variational equation

$$
\begin{aligned}
\delta \ni=\delta \int_{S} & \left\{T_{1}\left(\frac{\partial w}{\partial \theta}\right)^{2}+2 B^{-1} T_{12} \frac{\partial w}{\partial \alpha} \frac{\partial w}{\partial \theta}+B^{-1} T_{2}\left(\frac{\partial w}{\partial \alpha}\right)^{2}+\right. \\
& +K\left[\varepsilon_{1}^{2}+\varepsilon_{2}^{2}+2 v \varepsilon_{1} \varepsilon_{2}+2(1-v) \varepsilon_{12}^{2}\right]+ \\
& \left.+D\left[\chi_{1}^{2}+\chi_{2}^{2}+2 v \chi_{1} \chi_{2}+2(1-v) \chi_{12}^{2}\right]\right\} B d \alpha d \theta=0,
\end{aligned}
$$

where $\ni$ is the potential energy of deformation of the shell; $B=r \sin \theta$, $K=E h /\left(1-v^{2}\right), \quad D=E h^{3} / 12\left(1-v^{2}\right) ; \quad h$ is shell thickness; $S$ is the surface of the shell; $v$ is Poisson's ratio; $E$ is elastic modulus; $T_{i j}$ is efforts; $\varepsilon_{i j}$ is deformation; $\chi_{i j}$ is curvature; $\alpha, \theta$ are the coordinates.

Based on the proposed computational algorithm for the projectioniterative modification of the LVM, a calculation program for the PC was developed. The problem of loading a spherical shell reinforced by a ring 
with two identical dies with different coverage angles is investigated. In the calculations, a critical parameter $\lambda_{*}=P_{*} / E h^{2}$ was determined depending on the stiffness parameter of the system $i=E I E_{k}^{-1}\left(R \sin \vartheta_{S}\right)^{-4} \cdot 10^{7}$ and $\vartheta_{S}\left(P_{*}\right.$ is the critical force of stability loss; $E, E_{k}$ are the elastic modulus of the shell and ring; $\vartheta_{s}$ is the spherical coordinate of the edge of the shell; $R$ is the radius of the shell; $I$ is the moment of inertia of the ring).

In Fig. 2a shows the calculated dependence $\lambda_{1^{*}}$ on the parameter $i$ for a given $\vartheta_{s}\left(\vartheta_{S}=50^{\circ}\right)$. In Fig. $2 \mathrm{~b}$ dependence $\lambda_{2^{*}}$ on $\vartheta_{S}$ for a given $i$ $(i=5)$. The circles indicate the averaged values of the critical forces obtained in the experiment. In Fig. 2a, the results for 25 are averaged, in Fig. $2 \mathrm{~b}$ for 14 trials. The ranges of the parameters of the tested shells $R / h=400 \div 800, \vartheta_{S}=40 \div 60^{\circ}, i=2,5 \div 9$. The shells were loaded with two identical dies of short length (Fig. 1a).

High-speed filming showed that with a loss of stability, local dents initially have a circular shape, which in the experiment performed turned into an elliptical shape (such shapes are shown in Fig. 1).

We point out the possibility of considering optimization problems of choosing design parameters and dies that minimize critical load. This is done when constructing the calculated dependence $\lambda *$ for the indicated parameters (for the Winkler model, such a parameter characterizing the stiffness properties of loading dies is the bed coefficient).

The refinement of the calculation algorithm by applying the projection-iterative modification of the LVM led to a decrease in the estimated time by $4-5$ times in the calculation of critical forces. This was noted in $[1,2]$.

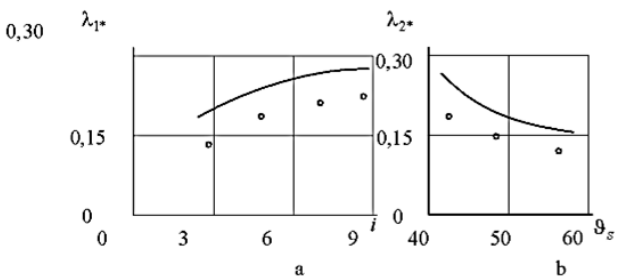

Fig. 2. The calculated dependences of critical parameters on the stiffness parameter of the support ring (a) and the spherical coordinate of the edge of the spherical shell (b) and averaged critical effort values 


\section{CONCLUSIONS}

The projection-iterative modification of the local variations method is especially effective for problems in which the introduction of a grid with a variable step is advisable. Such problems in the mechanics of a deformable solid include those in which a local stress concentration takes place. Important for priority branches of technology (rocket and space, antenna technology, etc.) are the problems of stability of shells under local loading. The features of such problems are shown by the example of the stability of spherical shells under local edge loading by dies, implemented through a force ring. The shape of the wave formation of the shell in this case is local in the zone of maximum stresses and depends on the structural and stiffness parameters of the system. The task in this case is to minimize the functional, which depends on the efforts of the subcritical stress-strain state of the shell and is replaced by the sum of the steps of the grid, which is automatically condensed in the stress concentration zone.

The developed projection-iterative modifications of the method of local variations allow significantly (for the problems considered, 4-5 times) to reduce the time of computer calculations. The values of the critical forces and the configurations of the stability waveforms obtained in the calculation are confirmed by the results of experimental data.

\section{SUMMARY}

Projection-iterative modification of the local variations method for solving variational problem with a quadratic functional is proposed. The convergence theorem is formulated. Application of the proposed modification to the problems of local stability of spherical shells under a substantially inhomogeneous stress state, which is caused by local edge loading, is considered. These tasks are important for the construction of space rocket, antenna technology, etc.

The developed projection-iterative modifications of the local variations method are effective and can significantly (more than 4 times) reduce the time of computer calculations. Numerical results are confirmed by experimental data. 


\section{REFERENCES}

1. E. L. Hart, "Projection-iterative schemes for implementing the method of local variations in problems for mechanics of deformable solid," in Proc. Int. Scientific Conf. Modern Probl. Mech., Lviv: LNU, 2009, P. 68.

2. E. Hart and V. Hudramovich, "Projection-iterative modifications of the local variation method and aspects of their application in problems for shells local stability," in Proc. Int. Scientific Conf. Modern Probl. Mech. and Math., Lviv: IPPMM NAS of Ukraine, 2008, vol. 3, pp. 18-20.

3. M. A. Krasnoselskii, G. M. Vainikko, P. P. Zabreiko, et al. Approximate Solution of Operator Equations, Moscow: Nauka, 1969.

4. V. I. Mossakovskii, V. S. Hudramovich, and E. M. Makeev, Contact Problems of Theory of Shells and Bars, Moscow: Mashinostrojenie, 1978.

5. Sh. A. Mukhamediev, L. V. Nikitin, and S. L. Jung, "Application of the modified method of local variations in problems of nonlinear fracture mechanics," Izv. USSR Academy of Sciences. Solid Mechanics, 1976, no. 1, pp. 76-83.

6. Kh. M. Mushtari and K. Z. Galimov, Nonlinear Theory of Elastic Shells, Kazan': Tatknigoizdat, 1957.

7. F. L. Chernous'ko and N. V. Banichuk, Variational Problems of Mechanics and Control, Moscow: Nauka, 1973.

8. E. L. Hart, "Projection-iterative version of the pointwise relaxation method," J. Math. Sci., 2010, vol. 167, no. 1, pp. 76-88.

9. E. L. Hart and V. S. Hudramovich, "Projection-iterative schemes for realization of the finite element method in problems of deformation of plates with holes and inclusions," J. Math. Sci., 2014, vol. 203, no. 1, pp. 55-69.

10.E. L. Hart and V.S. Hudramovich, "Projection-iterative modification of the method of local variations for problems with a quadratic functional," J. Appl. Math. and Mechanics, 2016, vol. 80, iss. 2, pp. 156-163.

11.V. S. Hudramovich, "Contact mechanics of shell structures under local loading," Int. Appl. Mech., 2009, vol. 45, no. 7, pp. 708-729. 
12.R. Kluge, "Ein Projektions-iterationsverfahren bei Fixpunkt problemen und Gleichungen mit monothonen Operatoren," Monatsber. Detsch. Akad. Wiss. Berlin, 1969, vol. 11, no. 8-9, pp. 599-609.

\section{Information about the authors:}

Hart E. L.,

Dr. Sci. (Phys.-Math.),

Professor at the Department of Theoretical and Computer Mechanics,

Oles Honchar Dnipro National University 72, Gagarin ave., Dnipro, 49010, Ukraine

Hudramovich V. S., Dr. Sci. (Tech.),

Corresponding member of the NAS of Ukraine, Professor, Head of the Department of Strength and Reliability of Mechanical Systems, Institute of Technical Mechanics of the Ukrainian National Academy of Sciences and State Space Agency of Ukraine 15, Leshko-Popel str., Dnipro, 49005, Ukraine 